Mathematical Modelling and Analysis

Volume 21 Number 3, May 2016, 319-335

http://dx.doi.org/10.3846/13926292.2016.1157835

(C) Vilnius Gediminas Technical University, 2016
Publisher: Taylor\&Francis and VGTU

http://www.tandfonline.com/TMMA

ISSN: $1392-6292$

eISSN: 1648-3510

\title{
Two-Dimensional Coupled Solution for Thermoelastic Beams via Generalized Dual-Phase-Lags Model
}

\author{
Ashraf M. Zenkour ${ }^{a, b}$ \\ ${ }^{a}$ Department of Mathematics, Faculty of Science, King Abdulaziz University \\ P.O. Box 80203, 21589 Jeddah, Saudi Arabia \\ ${ }^{b}$ Department of Mathematics, Faculty of Science, Kafrelsheikh University \\ 33516 Kafr El-Sheikh, Egypt \\ E-mail(corresp.): zenkour@kau.edu.sa \\ E-mail: zenkour@sci.kfs.edu.eg
}

Received June 8, 2015; revised February 19, 2016; published online May 15, 2016

\begin{abstract}
The generalized thermoelastic problem of a thick-walled simply-supported beam subjected to different applied heat source and mechanical loads at its surfaces is studied. The thermoelastic coupling differential equations of motion of the beam are established. The generalized thermoelasticity based on the dual-phase-lags (DPLs) theory is considered to treat this problem. An exact 2-D coupled solution is presented to deduce analytical expressions for the temperature, displacements and stresses. The time-harmonic motion behavior as well as the thermal and mechanical conditions at the bounded faces of the beam is used for this purpose. The effect of the DPLs on the field quantities against the axial and normal directions of the beam under thermomechanical loads is discussed. Final investigations to various thermoelastic models are made.

Keywords: generalized thermoelasticity, dual-phase-lags, state-space approach, thermomechanical loads.
\end{abstract}

AMS Subject Classification: $74 \mathrm{Fxx}$.

\section{Introduction}

A literature review reveals that many generalized theories of thermoelasticity have been developed to study the behavior of thermoelastic structures. These theories can be classified in different models, such as the theory of coupled thermoelasticity (CTE) [3], the Lord and Shulman (L-S) theory [13], the Green and Lindsay (G-L) theory [8], the Green and Naghdi (G-N) theory $[9,10,11]$ as well as the Tzou dual-phase-lag (DPL) thermoelasticity theory [21, 22, 23]. To the author's best knowledge, only a few authors have presented the exact two-dimensional solution of the generalized thermoelastic beam problem up to present time. 
Many investigators have used different forms of the normal mode analysis to obtain the exact analytical solutions of various thermoelastic problems $[1,8,9,10,11,19,21,22,23,28]$. Most of these publications have used the Laplace transformation to eliminate the time parameter. Sharma et al. [19] have applied the Hankel transform technique in the context of generalized theories of thermoelasticity to investigate the effect due to a time-harmonic normal point load and thermal source in a homogeneous isotropic thermoelastic, halfspace. Verma [24] has obtained the dispersion relations of thermoelastic waves by invoking continuity at the interface and boundary conditions on the surfaces of layered plate. Othman and Singh [16] have studied the equations of a 2-D problem in a micro-polar thermoelastic medium for a half-space whose surface is free and subjected to an instantaneous thermal point source. Jiangong and Tonglong [12] have used the G-N generalized thermoelastic theory without energy dissipation to investigate the propagation of thermoelastic waves in orthotropic spherical curved plates subjected to stress-free, isothermal boundary conditions. Mukhopadhyay and Kumar [15] have formulated the state-space approach to the problems of thermoelastic interactions on the basis of the G$\mathrm{N}$ theory of generalized thermoelasticity of type-III with energy dissipation. Prasad et al. [17] have dealt with the investigation of the propagation of harmonic plane waves with assigned frequency by employing the thermoelasticity theory with dual-phase-lags. Zhou et al. [29] have investigated the generalized coupled thermoelasticity based on the L-S theory by considering the transient thermoelastic response of functionally graded rectangular plates. Hyperbolic heat conduction problem is solved numerically by Čiegis [4].

The 3-D problem for a homogeneous, isotropic and thermoelastic halfspace subjected to a time-dependent heat have been considered by Sarkar and Lahiri [18]. They assumed that the boundary of the space is traction free and treat this problem in the context of G-N model II of thermoelasticity without energy dissipation. Lotfy [14] has presented the magneto-thermoelastic interactions in an isotropic homogeneous elastic half-space with two temperatures using mathematical methods under the purview of the CTE, L-S and G-L theories. Abbas [1] has established a 3-D model of the generalized thermoelasticity without energy dissipation under temperature-dependent mechanical properties. Recently, Zenkour [26] has presented a unified generalized thermoelasticity theory for the transient thermal shock plate problem in the context of G-L, L-S, and CTE theories.

El-Karamany and Ezzat [5] have proved the uniqueness and reciprocal theorems without the use of Laplace transforms for the dual-phase-lag thermoelasticity theory. Ezzat et al. [6] have constructed a new mathematical model of two-temperature magneto-thermoelasticity taking under consideration the fractional order dual-phase-lag heat conduction law.

The present article is concerned with the two-dimensional transient generalized thermoelastic problem for a beam subjected to thermal and thermomechanical loads at its faces. Based on the dual-phase-lags model $[2,27,28]$, the state equations are established by state-space method. The equations of the classical thermoelasticity theory, Lord and Shulman theory, and Green and Naghdi theory may be established as special cases of the DPLs theory. All 
expressions for temperature, displacements and stresses are presented. Numerical results showing the thermoelastic dynamic responses of the field quantities through the axial and thickness directions of the beam are presented.

\section{Governing equations}

Let us consider an elastic beam with dimensions of length $L(0 \leqslant x \leqslant L)$, width $b(-b / 2 \leqslant y \leqslant b / 2)$, and thickness $h(-h / 2 \leqslant z \leqslant h / 2)$, as shown in Figure 1. The $x$-axis is defined along the axis of the beam and the $y$ - and

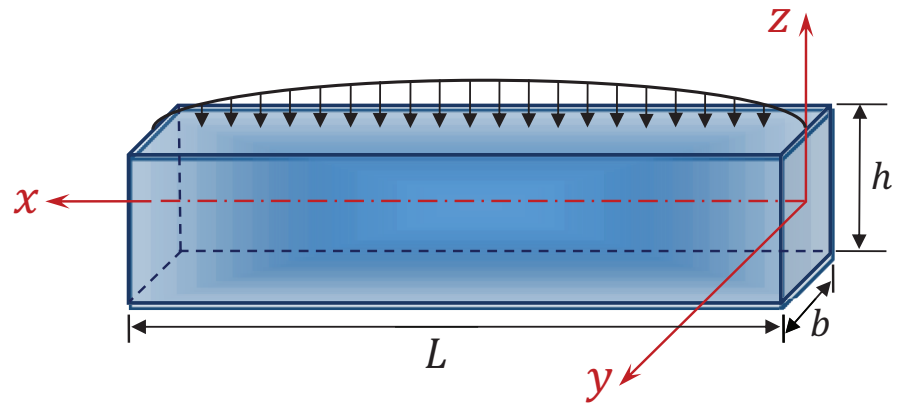

Figure 1. Schematic diagram of the beam.

$z$-axes correspond to the width and thickness, respectively. Let $u, v=0$, and $w$ denote the displacement components of a material point located at $(x, y, z)$ in the present beam in the $x, y$, and $z$ directions, respectively. The stress-strain relationships in the beam coordinates are written in the form:

$$
\left\{\begin{array}{l}
\sigma_{1} \\
\sigma_{3}
\end{array}\right\}=\left[\begin{array}{ll}
c_{11} & c_{13} \\
c_{13} & c_{33}
\end{array}\right]\left\{\begin{array}{l}
\varepsilon_{1}-\alpha \theta \\
\varepsilon_{3}-\alpha \theta
\end{array}\right\}, \quad \sigma_{5}=c_{55} \varepsilon_{5},
$$

where $\sigma_{j}$ and $\varepsilon_{j}$ are the stress and strain components, $\theta=T-T_{0}$ is the temperature increment of the resonator, in which $T(x, y, z)$ is the temperature distribution and $T_{0}$ is the environmental temperature, $\alpha$ is the linear thermal expansion coefficient. The elastic coefficients $c_{i j}$ are given, in terms of the engineering properties, by

$c_{11}=c_{33}=\frac{E(1-\nu)}{(1+\nu)(1-2 \nu)}, \quad c_{13}=\frac{\nu E}{(1+\nu)(1-2 \nu)}, \quad c_{55}=G=\frac{E}{2(1+\nu)}$,

where $E$ denotes the Young's modulus of elasticity, $\nu$ represents the Poisson's ratio and $G$ is the shear modulus. The strain-displacement relations are taken in the linear form as

$$
\varepsilon_{1}=\frac{\partial u}{\partial x}, \quad \varepsilon_{3}=\frac{\partial w}{\partial z}, \quad \varepsilon_{5}=\frac{\partial w}{\partial x}+\frac{\partial u}{\partial z} .
$$


The governing equations of motion can be presented as

$$
\begin{gathered}
c_{11} \frac{\partial^{2} u}{\partial x^{2}}+c_{55} \frac{\partial^{2} u}{\partial z^{2}}+\left(c_{13}+c_{55}\right) \frac{\partial^{2} w}{\partial x \partial z}-\alpha\left(c_{11}+c_{13}\right) \frac{\partial \theta}{\partial x}=\rho \frac{\partial^{2} u}{\partial t^{2}} \\
\left(c_{13}+c_{55}\right) \frac{\partial^{2} u}{\partial x \partial z}+c_{55} \frac{\partial^{2} w}{\partial x^{2}}+c_{33} \frac{\partial^{2} w}{\partial z^{2}}-\alpha\left(c_{13}+c_{33}\right) \frac{\partial \theta}{\partial z}=\rho \frac{\partial^{2} w}{\partial t^{2}}
\end{gathered}
$$

where $\rho$ is the material density of the beam.

In addition, the heat conduction in the context of generalized (non-Fourier) thermoelasticity for the above displacements in the absence of body forces, external loads and heat sources should be considered. The modified classical thermoelasticity model is given by Tzou theory in which the Fourier law is replaced by an approximation of the equation

$$
q\left(x, t+\tau_{q}\right)=-K \nabla T\left(x, t+\tau_{\theta}\right),
$$

where $q$ is the heat flux vector, $K$ is the thermal conductivity, and $\tau_{q}$ and $\tau_{\theta}$ represent the delay times. The delay time $\tau_{\theta}$ is said to be the phase-lag of the temperature gradient and the other delay time $\tau_{q}$ is called the phase-lag of the heat flux.

The above equation may be approximated by

$$
\left(1+\tau_{q} \frac{\partial}{\partial t}\right) q=-K\left(1+\tau_{\theta} \frac{\partial}{\partial t}\right) \nabla T
$$

where $0<\tau_{\theta}<\tau_{q}$. Then the heat conduction equation corresponding to the dual-phase-lag model proposed by Tzou in this case takes the form

$$
K\left(1+\tau_{\theta} \frac{\partial}{\partial t}\right) \nabla^{2} \theta+\left(1+\tau_{q} \frac{\partial}{\partial t}\right)\left(\rho Q^{*}\right)=\left(\delta+\tau_{q} \frac{\partial}{\partial t}\right)\left(\rho C^{e} \frac{\partial \theta}{\partial t}+\gamma T_{0} \frac{\partial e}{\partial t}\right)
$$

where $C^{e}$ is the specific heat per unit mass at constant strain, $e=\varepsilon_{k k}=\frac{\partial u}{\partial x}+\frac{\partial w}{\partial z}$ is the volumetric strain, $\gamma=E \alpha /(1-2 \nu), \delta$ is an unification parameter and $Q^{*}$ is the heat source. So, the final form of the thermal conduction equation for the present beam without heat source $\left(Q^{*}=0\right)$ is given as

$$
\left(1+\tau_{\theta} \frac{\partial}{\partial t}\right)\left(\frac{\partial^{2} \theta}{\partial x^{2}}+\frac{\partial^{2} \theta}{\partial z^{2}}\right)=\left(\delta+\tau_{q} \frac{\partial}{\partial t}\right)\left[\eta \frac{\partial \theta}{\partial t}+\frac{\gamma T_{0}}{K} \frac{\partial}{\partial t}\left(\frac{\partial u}{\partial x}+\frac{\partial w}{\partial z}\right)\right],
$$

where $\eta=\rho C^{e} / K$. The above equation describes the most generalized thermoelasticity theories. For example, the coupled dynamical thermoelasticity theory (CTE), the generalized thermoelasticity theories proposed by Lord and Shulman (L-S), Green and Naghdi (G-N) and dual-phase-lag (DPL) for different sets of values of phase-lags parameters $\tau_{q}, \tau_{\theta}$ and the unification parameter $\delta$ as follows:

CTE model: $\tau_{\theta}=\tau_{q}=0$ and $\delta=1$.

L-S model: $\tau_{\theta}=0, \tau_{q}=\tau_{0}\left(\tau_{0}\right.$ is the relaxation time) and $\delta=1$.

G-N model: $\tau_{\theta}=0, \delta=1, \tau_{q}=1$ and $K=K^{*}$ (the material constant characteristic of G-N theory).

DPL model: $\delta=1$ and $0<\tau_{\theta} \leq \tau_{q}$. 


\section{Solution of the problem}

In what follows we will use the following dimensionless variables and notations, for our convenience,

$$
\begin{aligned}
& \left\{x^{\prime}, z^{\prime}, L^{\prime}, h^{\prime}, u^{\prime}, w^{\prime}\right\}=\eta c\{x, z, L, h, u, w\}, \quad \sigma_{j}^{\prime}=\frac{1}{\rho c^{2}} \sigma_{j}, \quad \Theta^{\prime}=\frac{\Theta}{T_{0}}, \\
& \left\{t^{\prime}, \tau_{0}^{\prime}, \tau_{\theta}^{\prime}, \tau_{q}^{\prime}\right\}=\eta c^{2}\left\{t, \tau_{0}, \tau_{\theta}, \tau_{q}\right\}, \quad c^{2}=\frac{E}{\rho(1+\nu)(1-2 \nu)} .
\end{aligned}
$$

The equations of motion and heat equation become (dropping the prime for convenience)

$$
\begin{aligned}
& (1-\nu) \frac{\partial^{2} u}{\partial x^{2}}+\frac{1-2 \nu}{2} \frac{\partial^{2} u}{\partial z^{2}}+\frac{1}{2} \frac{\partial^{2} w}{\partial x \partial z}-\alpha T_{0} \frac{\partial \theta}{\partial x}=\frac{\partial^{2} u}{\partial t^{2}} \\
& \frac{1}{2} \frac{\partial^{2} u}{\partial x \partial z}+\frac{1-2 \nu}{2} \frac{\partial^{2} w}{\partial x^{2}}+(1-\nu) \frac{\partial^{2} w}{\partial z^{2}}-\alpha T_{0} \frac{\partial \theta}{\partial z}=\frac{\partial^{2} w}{\partial t^{2}} \\
& \left(1+\tau_{\theta} \frac{\partial}{\partial t}\right)\left(\frac{\partial^{2} \theta}{\partial x^{2}}+\frac{\partial^{2} \theta}{\partial z^{2}}\right)=\left(\delta+\tau_{q} \frac{\partial}{\partial t}\right)\left[\frac{\partial \theta}{\partial t}+\frac{\gamma}{\eta K} \frac{\partial}{\partial t}\left(\frac{\partial u}{\partial x}+\frac{\partial w}{\partial z}\right)\right] .
\end{aligned}
$$

The following simply-supported conditions are imposed at the edges of the beam:

$$
\sigma_{1}(x, z, t)=0, \quad w(x, z, t)=0, \quad \theta(x, z, t)=0, \quad \text { at } \quad x=0, L .
$$

Time-harmonic motion at angular frequency $\omega$ is considered here. So, the displacement and temperature components satisfying the above boundary conditions on the edges may be expressed as:

$$
\{u, w, \theta\}(x, z, t)=\{U(z) \cos (\xi x), W(z) \sin (\xi x), \Theta(z) \sin (\xi x)\} \mathrm{e}^{I \omega t},
$$

where $\xi=\pi / L$ is a mode number and $I=\sqrt{-1}$. Then, Eqs. (3.2), after some elementary manipulations, become

$$
\begin{aligned}
& \frac{\mathrm{d}^{2} U}{\mathrm{~d} z^{2}}=C_{1} U+C_{2} \Theta+C_{3} \frac{\mathrm{d} U}{\mathrm{~d} z}, \\
& \frac{\mathrm{d}^{2} W}{\mathrm{~d} x^{2}}=C_{4} W+C_{5} \frac{\mathrm{d} U}{\mathrm{~d} z}+C_{6} \frac{\mathrm{d} \Theta}{\mathrm{d} z}, \\
& \frac{\mathrm{d}^{2} \Theta}{\mathrm{d} x^{2}}=C_{7} U+C_{8} \Theta+C_{3} \frac{\mathrm{d} W}{\mathrm{~d} z},
\end{aligned}
$$

where the expressions $C_{k}(k=1, \cdots, 9)$ are given by

$$
\begin{aligned}
& C_{1}=\frac{2\left[\xi^{2}(1-\nu)-\omega^{2}\right]}{1-2 \nu}, \quad C_{2}=\frac{2 \xi \alpha T_{0}}{1-2 \nu}, \quad C_{3}=\frac{\xi}{1-2 \nu}, \\
& C_{4}=\frac{\xi^{2}(1-2 \nu)-2 \omega^{2}}{1-2 \nu}, \quad C_{5}=\frac{\xi}{2(1-\nu)}, \quad C_{6}=\frac{\alpha T_{0}}{1-\nu}, \\
& C_{7}=-\frac{\left(\delta+\tau_{q} I \omega\right) I \omega \gamma \xi}{\left(1+\tau_{\theta} I \omega\right) K \eta}, \quad C_{8}=\xi^{2}-\frac{K \eta C_{7}}{\gamma \xi}, \quad C_{9}=-\frac{C_{7}}{\xi}
\end{aligned}
$$


For the sake of convenience the state variables are introduced as follows [25] (see also $[6,7])$ :

$$
\begin{aligned}
& S_{1}=\Theta, \quad S_{2}=U, \quad S_{3}=W, \quad S_{4}=\frac{\mathrm{d} \Theta}{\mathrm{d} z}=\frac{\mathrm{d} S_{1}}{\mathrm{~d} z}, \\
& S_{5}=\frac{\mathrm{d} U}{\mathrm{~d} z}=\frac{\mathrm{d} S_{2}}{\mathrm{~d} z}, \quad S_{6}=\frac{\mathrm{d} W}{\mathrm{~d} z}=\frac{\mathrm{d} S_{3}}{\mathrm{~d} z} .
\end{aligned}
$$

Equations (13) may be reduced to the matrix form as

$$
\left\{\frac{\mathrm{d} S}{\mathrm{~d} z}\right\}=[A]\{S\}
$$

where the vector $\{S\}$ and the matrix $[A]$ are given, respectively, by

$$
S=\left\{\begin{array}{c}
S_{1} \\
S_{2} \\
\vdots \\
S_{6}
\end{array}\right\}, \quad[A]=\left[\begin{array}{cccccc}
0 & 0 & 0 & 1 & 0 & 0 \\
0 & 0 & 0 & 0 & 1 & 0 \\
0 & 0 & 0 & 0 & 0 & 1 \\
c_{8} & c_{7} & 0 & 0 & 0 & c_{9} \\
c_{2} & c_{1} & 0 & 0 & 0 & c_{3} \\
0 & 0 & c_{4} & c_{6} & c_{5} & 0
\end{array}\right]
$$

The general solution of Eq. (3.3) is available since $[A]$ has distinct eigenvalues and it is given by

$$
S=[R]\left[\begin{array}{cccc}
\mathrm{e}^{\lambda_{1} z} & & & 0 \\
& \mathrm{e}^{\lambda_{2} z} & & \\
& & \ddots & \\
0 & & & \mathrm{e}^{\lambda_{6} z}
\end{array}\right][R]^{-1} B
$$

where $B_{1}, B_{2}, \cdots, B_{6}$ are arbitrary unknown complex constants connected with the boundary conditions. Here, $\lambda_{k}, \lambda_{k+1}=-\lambda_{k}(k=1,3,5)$ and $[R]$ are the eigenvalues and the matrix of eigenvector of the coefficient matrix $[A]$ and $[R]^{-1}$ is the inverse of the matrix $[R]$. The eigenvalues $\lambda_{j}(j=1,6)$ and the elements of the matrix of eigenvector $[R]$ are generally complex valued. Equation (3.4) can alternatively be presented as

$$
S_{i}=\sum_{j=1}^{6} s_{i j} \mathrm{e}^{\lambda_{j} z} B_{j}, \quad j=1,2, \cdots, 6,
$$

where $s_{i j}=\lambda_{j} s_{(i-3) j},(i=4,5,6)$ and the other components of the expressions $s_{i j}(i=1,2,3)$ are given by

$$
S_{1 j}=1, \quad S_{2 j}=A_{4} \lambda_{j}^{4}+A_{5} \lambda_{j}^{2}+A_{6}, \quad S_{3 j}=\left(A_{7} \lambda_{j}^{4}+A_{8} \lambda_{j}^{2}+A_{9}\right) \lambda_{j} .
$$

In addition, the components of the coefficients $A_{i}(i=1,9)$ and $\lambda_{j}$ are given in Appendix A. 
The temperature $\theta$ and the displacements $u, w$ may be easily given from the above relations. That is

$$
\begin{aligned}
& \theta=\sin (\xi x) \mathrm{e}^{I \omega t} \sum_{j=1}^{6} s_{1 j} \mathrm{e}^{\lambda_{j} z} B_{j}, \quad u=\cos (\xi x) \mathrm{e}^{I \omega t} \sum_{j=1}^{6} s_{2 j} \mathrm{e}^{\lambda_{j} z} B_{j}, \\
& w=\sin (\xi x) \mathrm{e}^{I \omega t} \sum_{j=1}^{6} s_{3 j} \mathrm{e}^{\lambda_{j} z} B_{j} .
\end{aligned}
$$

In addition, the temperature and the displacements and their first derivatives with respect to $z$ are used to obtain the stresses in the present beam as

$$
\begin{aligned}
& \sigma_{1}=\sin (\xi x) \mathrm{e}^{I \omega t} \sum_{j=1}^{6}\left[-\xi(1-\nu) s_{2 j}-\alpha T_{0} s_{1 j}+\lambda_{j} \nu s_{3 j}\right] \mathrm{e}^{\lambda_{j} z} B_{j}, \\
& \sigma_{3}=\sin (\xi x) \mathrm{e}^{I \omega t} \sum_{j=1}^{6}\left[-\xi \nu s_{2 j}-\alpha T_{0} s_{1 j}+\lambda_{j}(1-\nu) s_{3 j}\right] \mathrm{e}^{\lambda_{j} z} B_{j}, \\
& \sigma_{5}=\frac{1-2 \nu}{2} \cos (\xi x) \mathrm{e}^{I \omega t} \sum_{j=1}^{6}\left[\xi s_{3 j}+\lambda_{j} s_{2 j}\right] \mathrm{e}^{\lambda_{j} z} B_{j} .
\end{aligned}
$$

To obtain the displacements, temperature, and stresses of the beam, thermal and mechanical boundary conditions must be satisfied according to the following two cases:

Case 1: Pure thermal conditions

The thermal conditions are taken as

$$
\theta\left(x, \frac{h}{2}, t\right)=T_{0} \sin (\xi x) \mathrm{e}^{I \omega t},\left.\quad \frac{\partial \theta}{\partial z}\right|_{z=-\frac{h}{2}}=0,
$$

where $\frac{\partial \theta}{\partial z}$ denotes the normal components of the heat flux vector. Also, the mechanical boundary conditions on the face surfaces of the beam have the form

$$
\sigma_{3}\left(x, \pm \frac{h}{2}, t\right)=\sigma_{5}\left(x, \pm \frac{h}{2}, t\right)=0 .
$$

\section{Case 2: Thermomechanical conditions}

The thermal conditions are the same as in Eq. (3.7) while the mechanical boundary conditions on the face surfaces of the beam will be

$$
\sigma_{3}\left(x, \frac{h}{2}, t\right)=\sigma_{0} \sin (\xi x) \mathrm{e}^{I \omega t}, \quad \sigma_{3}\left(x,-\frac{h}{2}, t\right)=\sigma_{5}\left(x, \pm \frac{h}{2}, t\right)=0,
$$

where $\sigma_{0}$ represents the intensity of the applied load at the upper face surface of the beam. For the comparison purpose, we can take $\sigma_{0}$ equals to the unity.

The results of the present thermomechanical loaded beam will be considered in the following section. Equations (3.5) and (3.6) in conjunction with the boundary conditions of Eqs. (3.7) and (3.8) or (3.9) yield a determination of the arbitrary constants. 


\section{Numerical results}

The thermoelastic coupling effect is presented here to get the temperature, displacements and stresses in the present beam. The material parameters used here are due to the physical data of magnesium [20]:

$$
\begin{aligned}
& \rho=1.74 \times 10^{3} \mathrm{~kg} \mathrm{~m}^{-3}, \quad E=4.212 \times 10^{10} \mathrm{~N} \mathrm{~m}^{-2}, \quad G=1.639 \times 10^{10} \mathrm{~N} \mathrm{~m}^{-2}, \\
& \nu=0.285, \quad K=170 \mathrm{~W} \mathrm{~m}^{-1} \mathrm{deg}^{-1}, \quad \gamma=2.68 \times 10^{6} \mathrm{~W} \mathrm{~m}^{-2} \mathrm{deg}^{-1}, \\
& C^{e}=1040 \mathrm{~J} \mathrm{~kg} \mathrm{deg}^{-1}, \quad T_{0}=25^{\circ} \mathrm{C} .
\end{aligned}
$$

The length-to-thickness ratio of the beam is fixed as $L / h=5$ and the angular frequency $\omega=2$. All plots are prepared by using the real values of the dimensionless variables defined in Eq. (3.1) for a wide range of beam length and thickness. Once again, the directions of the beam are given in terms of the length and thickness of the beam, that is $x^{\prime}=x / l$ and $z^{\prime}=z / h$ (the prime will be dropping in the figures for convenience). The computations are carried out for one value of time, namely $t=0.2$ and various values of the delay time parameters $\tau_{\theta}$ and $\tau_{q}$. Figures 2-13 compared the results obtained for temperature, displacements, and stresses against the $x$ and $z$ directions for beams under various loads. The graphs represent curves predicted by the CTE, L-S and G-N models of thermoelasticity obtained as special cases of the present general the DPL model. The results of the CTE model $\left(\tau_{\theta}=\tau_{q}=0, \delta=1\right)$, the L-S model $\left(\tau_{\theta}=0, \tau_{q}=0.1, \delta=1\right)$, the G-N model $\left(\tau_{\theta}=0, \tau_{q}=0.1, \delta=0\right)$, and the DPL model $\left(\tau_{\theta}=0.05<\tau_{q}=0.1, \delta=1\right)$, are all presented.

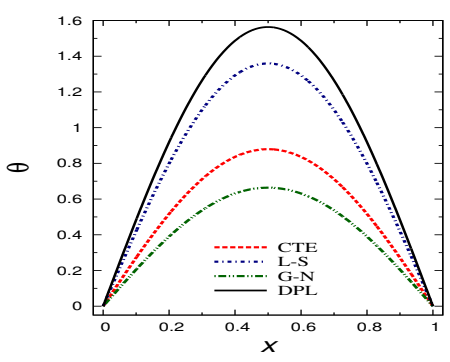

(a)

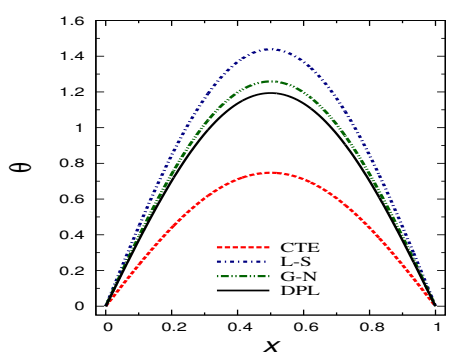

(b)

Figure 2. The temperature distributions in the axial direction for beams under (a) thermal load and (b) thermomechanical load.

Figure 2 shows the variation of the dimensionless temperature $\theta$ along the axial direction at the bottom surface $z=-0.5$ of the beam under $(a)$ thermal load and $(b)$ thermomechanical load. The behaviors of all models may take different shapes. The maximum temperature occurs at the center of the beam. The G-N model gives the smallest temperature for the beam under thermal load only and the CTE model gives the smallest temperature for the beam under thermomechanical load. However, the DPL model gives the largest one 
for the first case and the L-S model gives the largest one for the second case. The temperatures due to the DPL and CTE models are decreased when the mechanical load is added. This is not the same for the temperatures due to the L-S and G-N models.

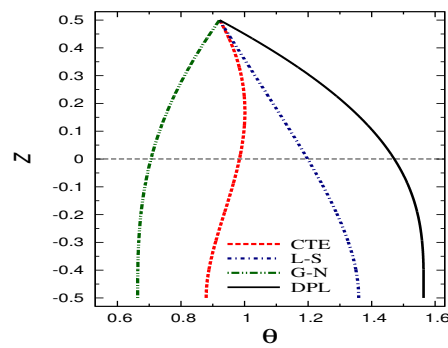

(a)

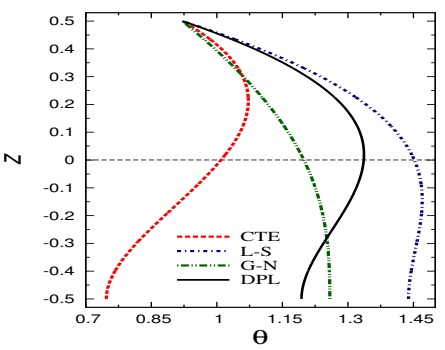

(b)

Figure 3. The temperature distributions in the thickness direction for beams under (a) thermal load and (b) thermomechanical load.

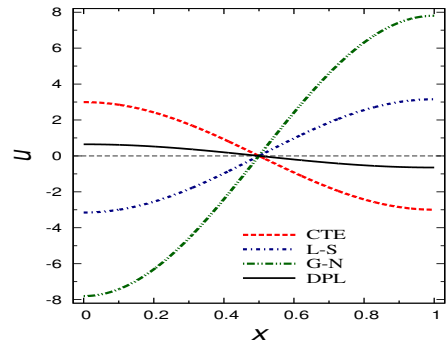

(a)

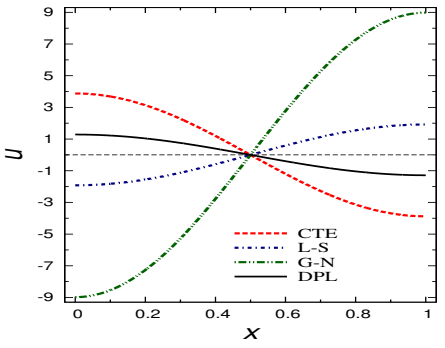

(b)

Figure 4. The axial displacement distributions in the axial direction for beams under (a) thermal load and (b) thermomechanical load.

Figure 3 shows the through-the-thickness variation of the dimensionless temperature at $x=0.5$ of the beam under $(a)$ thermal load and $(b)$ thermomechanical load. The maximum temperature occurs at the bottom face of the beam under thermomechanical load according to the G-N, L-S and DPL models. The CTE model gives minimum temperatures on the bottom beam face due to thermomechanical load. The temperature due to the G-N model is decreasing through the beam thickness under thermal load only. In fact, the DPL and L-S models may be suitable to represent the temperature distribution.

Figure 4 shows the variation of the dimensionless axial displacement $u$ along the axial direction at the mid-plane $z=0$ of the beam under (a) thermal load and (b) thermomechanical load. The axial displacement vanishes at the center of the beam. The G-N model may be unsuitable to treat with this displacement 
along the axial direction. It always gives absolute high displacements. The smallest axial displacements are given due to the DPL model for the two cases of the beam load. The axial displacement may be little enlarged for beams under thermomechanical load. However, the behaviors of the DPL, CTE and the L-S models are unchanged for the two cases.

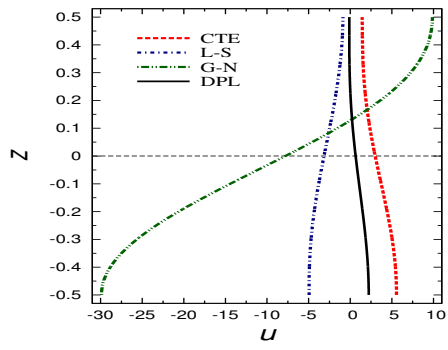

(a)

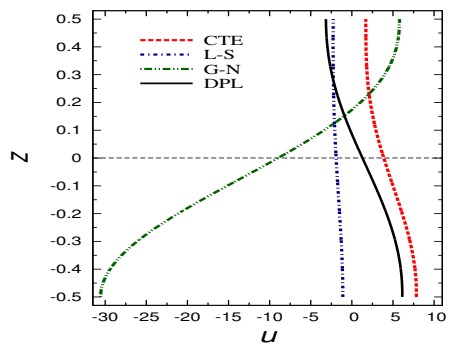

(b)

Figure 5. The axial displacement distributions in the thickness direction for beams under (a) thermal load and (b) thermomechanical load.

Figure 5 shows the through-the-thickness variation of the axial displacement $u$ at the first edge $x=0$ of the beam under various loads. The G-N model may be still unsuitable for the two cases. Other models give closed axial displacement through-the-thickness of the beam. The DPL model is closed with the L-S one through the upper half-plane of the beam and with the CTE model through the bottom half-plane.

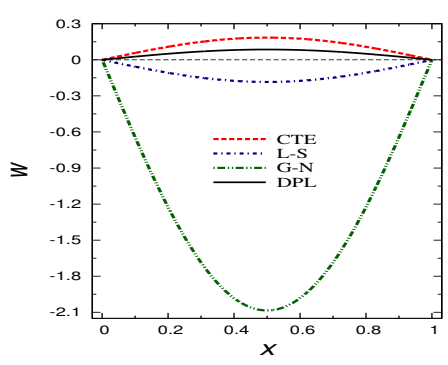

(a)

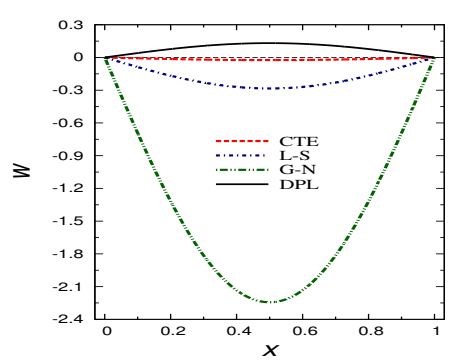

(b)

Figure 6. The transverse deflection distributions in the axial direction for beams under (a) thermal load and (b) thermomechanical load.

Figure 6 shows the variation of the transverse displacement $w$ along the axial direction at the middle surface $z=0$. Each model gives different behavior of $w$ along the axial direction of the beam. The maximum (minimum) deflection occurs at the center of the beam for the CTE and DPL models (G-N and L-S models) in the first case. However, the maximum deflection occurs at 


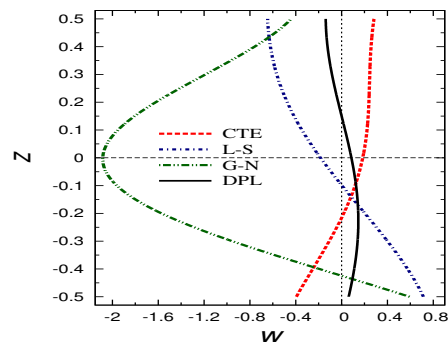

(a)

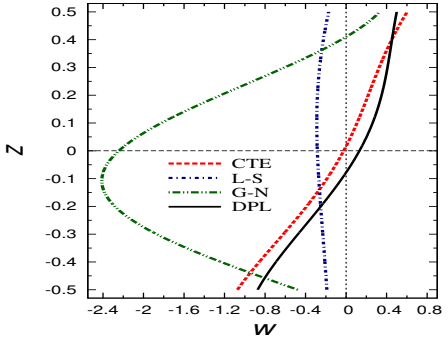

(b)

Figure 7. The transverse deflection distributions in the thickness direction for beams under (a) thermal load and (b) thermomechanical load.

the center of the beam for the DPL model only in the second case. It is to be noted that, the deflection due to the CTE model may be vanished in the second case. Figure 7 shows the through-the-thickness variation of the dimensionless transverse displacement $w$ at $x=0.5$. The deflections due to the CTE, L-S and DPL models are closed to each other. The G-N model may be having the same behavior through-the-thickness of the beam and still unsuitable for the two cases.

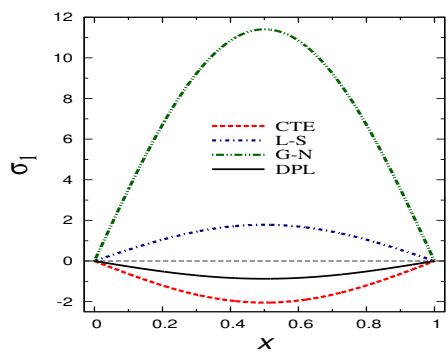

(a)

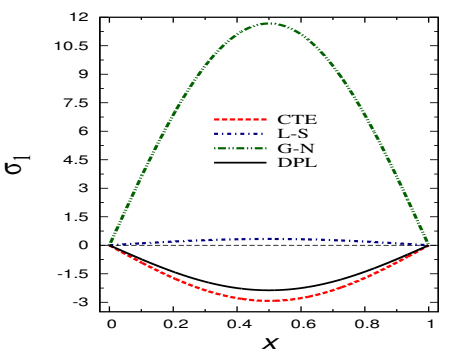

(b)

Figure 8. The axial normal stress distributions in the axial direction for beams under (a) thermal load and (b) thermomechanical load.

Figure 8 shows the variation of the dimensionless axial stress $\sigma_{1}$ along the axial direction at the bottom surface $z=-0.5$. The absolute maximum axial stress $\sigma_{1}$ occurs at the center of the beam. For the two loaded beams, the axial stress $\sigma_{1}$ of the G-N and L-S models still positive while those of the DPL and CTE models still negative along the axial direction. The G-N gives the highest axial stresses along the axis of the beam in the two cases. Figure 9 shows the through-the-thickness variation of the dimensionless axial stress $\sigma_{1}$ at the center of the beam $x=0.5$. The G-N model may be failed to get accurate axial stress. Other models may be closed to each other. The DPL model gives tensile 




(a)

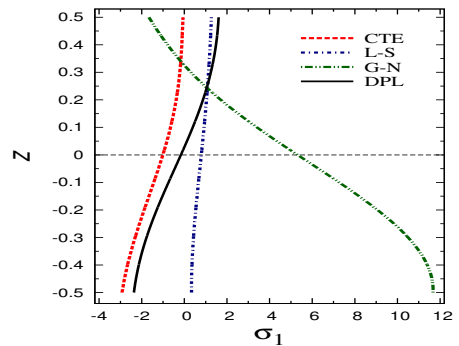

(b)

Figure 9. The axial normal stress distributions in the thickness direction for beams under (a) thermal load and (b) thermomechanical load.

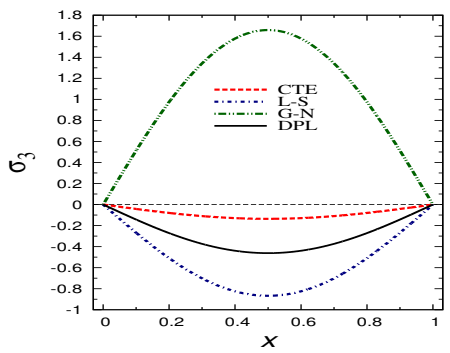

(a)

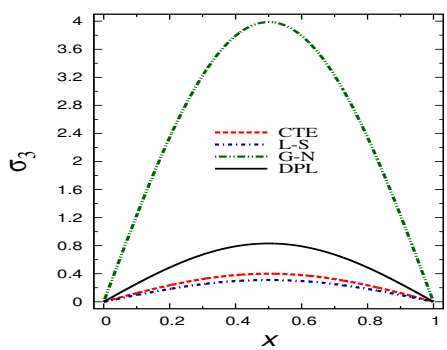

(b)

Figure 10. The transverse normal stress distributions in the axial direction for beams under (a) thermal load and (b) thermomechanical load.

(compression) stresses at the upper (bottom) surface of the beam. However, the L-S (CTE) model gives tensile (compressive) stresses through-the-thickness of the beam.

Figure 10 shows the variation of the dimensionless normal stress $\sigma_{3}$ along the axial direction at $z=0$. The G-N model gives tensile transverse normal stress in the two cases of the thermal and thermomechanical loads. The stresses due to other models are changed from compressive stresses in the thermal loaded beam to tensile stresses in the thermomechanical loaded beam. Figure 11 shows the through-the-thickness variation of the dimensionless normal stress $\sigma_{3}$ at the center of the beam $x=0.5$ All models are very sensitive to the variation of the used load. The G-N model gives the greatest tensile stress above the mid-plane of the beam (at $z=0.15$ ) while the L-S model gives the smallest compressive stress at $z=-0.1$ of the thermally loaded beam only. Figure 12 shows the variation of the dimensionless transverse shear stress $\sigma_{5}$ along the axial direction at $z=0$. The shear stresses are vanished at the center of axial direction according to all models. The G-N model may be failed to get accurate shear stress for beams subjected to different loads. It is to be noted that the L-S models gives 


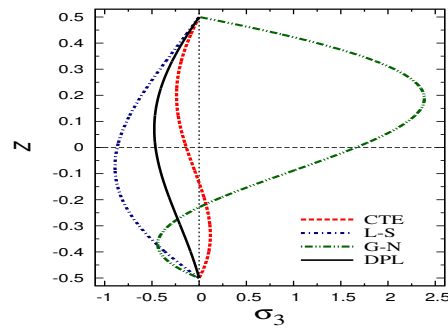

(a)

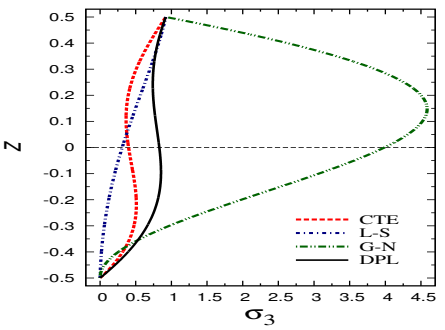

(b)

Figure 11. The transverse normal stress distributions in the thickness direction for beams under (a) thermal load and (b) thermomechanical load.

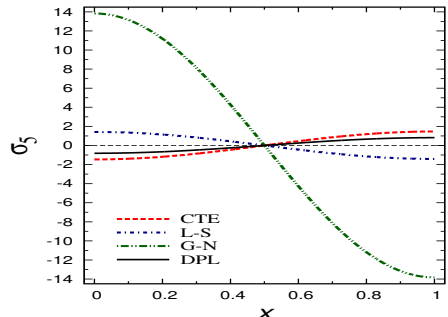

(a)

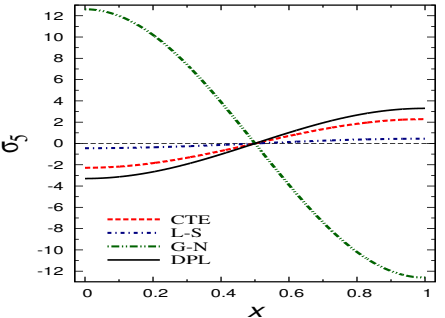

(b)

Figure 12. The transverse shear stress distributions in the axial direction for beams under (a) thermal load and (b) thermomechanical load.

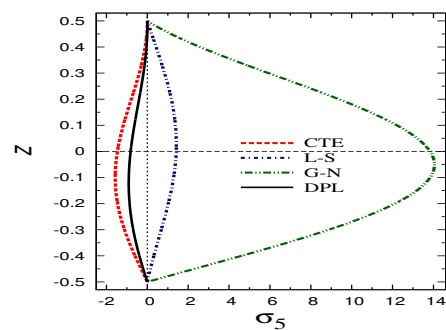

(a)

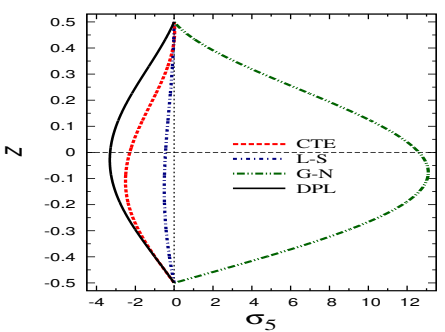

(b)

Figure 13. The transverse shear stress distributions in the thickness direction for beams under (a) thermal load and (b) thermomechanical load.

the smallest shear stress for thermomechanical loaded beams. Figure 13 shows the through-the-thickness variation of the dimensionless transverse shear stress $\sigma_{5}$ at the first edge $x=0$ of the beam. The behavior of the transverse shear stresses for the two cases may be the same. Just the first case enlarges the 
shear stresses with little changes in the behavior of the shear stress via the L-S model. Also, the CTE and DPL models replace their positions.

\section{Conclusions}

The exact presentations of the temperature, displacements and stresses in the axial and thickness directions of a generalized thermoelastic beam are considered in this article. The model of generalized thermoelasticity with dual-phaselags is constructed and other known thermoelastic models may be considered as special cases. The exact $2 \mathrm{D}$ general solution is applied to the present beam subjected to various heating source or thermomechanical load. The comparisons are shown along the axial and thickness directions of the beam. The field quantities are very sensitive to the applied load. Values of the field quantities induced by the thermomechanical load may be differ of those due to the thermal load. The method used here may be applicable to a wide range of problems in thermodynamics and thermoelasticity. The numerical results presented here may be considered as more general in the sense that they include the exact analysis of different field quantities. It is concluded from the graphical results presented here that the effect of dual-phase-lag parameters plays a significant role on all the physical quantities. Some of models may be failed to treat the thermoelastic response of many structures. This means that, the investigators may be not restricting their attentions to a specific thermoelastic generalized theory.

\section{References}

[1] I.A. Abbas. Eigenvalue approach in a three-dimensional generalized thermoelastic interactions with temperature-dependent material properties. Comput. Math. Appl., 68(12):2036-2056, 2014. http://dx.doi.org/10.1016/j.camwa.2014.09.016.

[2] I.A. Abbas and A.M. Zenkour. Dual-phase-lag model on thermoelastic interactions in a semi-infinite medium subjected to a ramptype heating. J. Comput. Theor. Nanosci., 11(3):642-645, 2014. http://dx.doi.org/10.1166/jctn.2014.3407.

[3] M.A. Biot. Thermoelasticity and irreversible thermodynamics. J. Appl. Phys., 27:240-253, 1956. http://dx.doi.org/10.1063/1.1722351.

[4] R. Čiegis. Numerical solution of hyperbolic heat conduction equation. Math. Model. Analys., 14(1):11-24, 2009. http://dx.doi.org/10.3846/13926292.2009.14.11-24.

[5] A.S. El-Karamany and M.A. Ezzat. On the dual-phase-lag thermoelasticity theory. Mecc., 49(1):79-89, 2014. http://dx.doi.org/10.1007/s11012-013-9774-z.

[6] M.A. Ezzat, A.S. El-Karamany and S.M. Ezzat. Two-temperature theory in magneto-thermoelasticity with fractional order dualphase-lag heat transfer. Nucl. Eng. Design, 252:267-277, 2012. http://dx.doi.org/10.1016/j.nucengdes.2012.06.012.

[7] M.A. Ezzat, M. I. Othman and A.S. El-Karamany. State space approach to two-dimensional generalized thermoviscoelasticity with one relaxation time. $J$. Therm. Stress, 25:295-316, 2002. 
[8] A.E. Green and K.A. Lindsay. Thermoelasticity. J. Elast., 2(1):1-7, 1972. http://dx.doi.org/10.1007/BF00045689.

[9] A.E. Green and P.M. Naghdi. A re-examination of the basic postulates of thermomechanics. Proc. Royal Soc., 432(1885):171-194, 1991. http://dx.doi.org/10.1098/rspa.1991.0012.

[10] A.E. Green and P.M. Naghdi. On undamped heat waves in an elastic solid. J. Thermal Stresses, 15(2):253-264, 1992. http://dx.doi.org/10.1080/01495739208946136.

[11] A.E. Green and P.M. Naghdi. Thermoelasticity without energy dissipation. J. Elast., 31(3):189-209, 1993. http://dx.doi.org/10.1007/BF00044969.

[12] Y. Jiangong and X. Tonglong. Generalized thermoelastic waves in spherical curved plates without energy dissipation. Acta Mech., 212(1):39-50, 2010. http://dx.doi.org/10.1007/s00707-009-0238-4.

[13] H.W. Lord and Y. Shulman. A generalized dynamical theory of thermoelasticity. Journal of the Mechanics and Physics of Solids, 15(5):299-309, 1967. http://dx.doi.org/10.1016/0022-5096(67)90024-5.

[14] Kh. Lotfy. Two temperature generalized magneto-thermoelastic interactions in an elastic medium under three theories. Appl. Math. Comput., 227:871-888, 2014. http://dx.doi.org/10.1016/j.amc.2013.11.063.

[15] S. Mukhopadhyay and R. Kumar. State-space approach to thermoelastic interactions in generalized thermoelasticity type III. Arch. Appl. Mech., 80(8):869-881, 2010. http://dx.doi.org/10.1007/s00419-009-0345-7.

[16] M.I.A. Othman and B. Singh. The effect of rotation on generalized micropolar thermoelasticity for a half-space under five theories. Int. J. Solids Struct., 44(9):2748-2762, 2007. http://dx.doi.org/10.1016/j.ijsolstr.2006.08.016.

[17] R. Prasad, R. Kumar and S. Mukhopadhyay. Propagation of harmonic plane waves under thermoelasticity with dual-phase-lags. Int. J. Eng. Sci., 48(12):2028-2043, 2010. http://dx.doi.org/10.1016/j.ijengsci.2010.04.011.

[18] N. Sarkar and A. Lahiri. A three-dimensional thermoelastic problem for a half-space without energy dissipation. Int. J. Eng. Sci., 51:310-325, 2012. http://dx.doi.org/10.1016/j.ijengsci.2011.08.005.

[19] J.N. Sharma, R.S. Chauhan and R. Kumar. Time-harmonic sources in a generalized thermoelastic continuum. J. Therm. Stresses, 23(7):657-674, 2000. http://dx.doi.org/10.1080/01495730050130048.

[20] J.N. Sharma and D. Kaur. Generalized thermoelastic plane harmonic waves in materials with voids. Int. J. Appl. Mech. Eng., 13:1019-1043, 2008.

[21] D.Y. Tzou. Experimental support for the lagging behavior in heat propagation. J. Thermophys. Heat Transfer, 9:686-693, 1995.

[22] D.Y. Tzou. A unified approach for heat conduction from macro- to micro-scales. J. Heat Transfer, 117(1):8-16, 1995. http://dx.doi.org/10.1115/1.2822329.

[23] D.Y. Tzou. Macro-to-microscale heat transfer: the Lagging behavior, Washington. DC, Taylor Francis, 1996.

[24] K.L. Verma. On the propagation of waves in layered anisotropic media in generalized thermoelasticity. Int. J. Eng. Sci., 40(18):2077-2096, 2002. http://dx.doi.org/10.1016/S0020-7225(02)00030-7. 
[25] A.M. Zenkour. Three-dimensional elasticity solutions for uniformly loaded crossply laminates and sandwich plates. J. Sand. Struct. Mater., 9(3):213-238, 2007. http://dx.doi.org/10.1177/1099636207065675.

[26] A.M. Zenkour. Three-dimensional thermal shock plate problem within the framework of different thermoelasticity theories. Compos. Struct., 132:1029-1042, 2015. http://dx.doi.org/10.1016/j.compstruct.2015.07.013.

[27] A.M. Zenkour and A.E. Abouelregal. The nonlocal dual phase lag model of thermoelastic nanobeam subjected to a sinusoidal pulse heating. Int. J. Compu. Meth. Eng. Sci. Mech., 16(1):53-73, 2015. http://dx.doi.org/10.1080/15502287.2014.976676.

[28] A.M. Zenkour, D.S. Mashat and A.E. Abouelregal. The effect of dual-phaselag model on reflection of thermoelastic waves in a solid half space with variable material properties. Acta Mech. Solida Sinca, 26:659-670, 2013. http://dx.doi.org/10.1016/S0894-9166(14)60009-4.

[29] F-X. Zhou, S-R. Li and Y-M. Lai. Three-dimension analysis for transient coupled thermoelastic response of a functionally graded rectangular plate. J. Sound Vib., 330:3990-4001, 2011. http://dx.doi.org/10.1016/j.jsv.2011.03.015.

\section{Appendix A}

The components of the coefficients $A_{i}$ appeared in Eq. (3.6) are given by

$$
\begin{aligned}
A_{1}= & C_{1}+C_{4}+C_{8}+C_{3} C_{5}+C_{6} C_{9}, \\
A_{2}= & -C_{1}\left(C_{4}+C_{8}+C_{6} C_{9}\right)+C_{2}\left(C_{7}+C_{5} C_{9}\right)-C_{3}\left(C_{5} C_{8}-C_{6} C_{7}\right)-C_{4} C_{8}, \\
A_{3}= & C_{4}\left(C_{1} C_{8}-C_{2} C_{7}\right), \quad A_{4}=C_{9} / \triangle \\
A_{5}= & -\frac{C_{9}\left(C_{4}+C_{8}+C_{3} C_{5}+C_{6} C_{9}\right)+C_{3} C_{7}}{\triangle}, \\
A_{6}= & -\frac{\left(C_{7}+C_{5} C_{9}\right)\left(C_{2} C_{9}-C_{3} C_{8}\right)-C_{4} C_{8} C_{9}}{\triangle}, \quad A_{7}=-\frac{C_{7}+C_{5} C_{9}}{C_{4} \triangle}, \\
A_{8}= & \frac{\left(C_{7}+C_{5} C_{9}\right)\left(C_{1}+C_{8}+C_{3} C_{5}+C_{6} C_{9}\right)+C_{4} C_{5} C_{9}}{C_{4} \triangle}, \\
A_{9}= & -\frac{\left(C_{7}+C_{5} C_{9}\right)\left(C_{1} C_{8}-C_{2} C_{7}+C_{3} C_{5} C_{8}+C_{1} C_{6} C_{9}-C_{3} C_{6} C_{7}-C_{2} C_{5} C_{9}\right)}{C_{4} \triangle} \\
& -\frac{C_{4} C_{9}\left(C_{5} C_{8}-C_{6} C_{7}\right)}{C_{4} \triangle},
\end{aligned}
$$

where $\triangle=C_{1} C_{5} C_{9}^{2}+C_{7} C_{9}\left(C_{1}-C_{4}-C_{3} C_{5}\right)-C_{3} C_{7}^{2}$.

The components of the coefficients $\lambda_{1}, \lambda_{3}$ and $\lambda_{5}$ appeared in Eq. (3.6) are given by

$$
\lambda_{1}=-\frac{\sqrt{I\left[(\sqrt{3}+I) A_{0}^{2 / 3}-4(\sqrt{3}-I)\left(A_{1}^{2}+3 A_{2}\right)-4 I A_{1} A_{0}^{1 / 3}\right]}}{2 \sqrt{3} A_{0}^{1 / 6}},
$$




$$
\begin{aligned}
& \lambda_{3}=-\frac{\sqrt{-I\left[(\sqrt{3}-I) A_{0}^{2 / 3}-4(\sqrt{3}+I)\left(A_{1}^{2}+3 A_{2}\right)+4 I A_{1} A_{0}^{1 / 3}\right]}}{2 \sqrt{3} A_{0}^{1 / 6}}, \\
& \lambda_{5}=-\frac{\sqrt{A_{0}^{2 / 3}+2 A_{1} A_{0}^{1 / 3}+4 A_{1}^{2}+12 A_{2}}}{\sqrt{6} A_{0}^{1 / 6}},
\end{aligned}
$$

where

$$
\begin{aligned}
A_{0}= & 36 A_{1} A_{2}+108 A_{3}+8 A_{1}^{2} \\
& +12 \sqrt{12 A_{1}^{3} A_{3}-3 A_{1}^{2} A_{2}^{2}+54 A_{1} A_{2} A_{3}-12 A_{2}^{3}+81 A_{3}^{2}} .
\end{aligned}
$$

